Western University

Scholarship@Western

Education Publications

Education Faculty

$10-17-2017$

\title{
Using Intraverbal Prompts to Increase Divergent Intraverbal Responses by a Child with Autism
}

Gabrielle Lee

Western University, glee329@uwo.ca

Wan-Chi Chou

National Changhua University of Education

Hua Feng

National Changhua University of Education

Follow this and additional works at: https://ir.lib.uwo.ca/edupub

Part of the Education Commons

Citation of this paper:

Lee, Gabrielle; Chou, Wan-Chi; and Feng, Hua, "Using Intraverbal Prompts to Increase Divergent Intraverbal Responses by a Child with Autism" (2017). Education Publications. 141.

https://ir.lib.uwo.ca/edupub/141 
Running head: DIVERGENT INTRAVERBAL RESPONSES

Using Intraverbal Prompts to Increase Divergent Intraverbal Responses by a Child with Autism

Lee, G. T., Chou, W., \& Feng, H. (2017). Using intraverbal prompts to increase divergent intraverbal responses by a child with autism. Behavioral Interventions, 32(4), 434-444. 


\begin{abstract}
We examined the effectiveness of intraverbal prompts to increase the number of divergent responses to categorical questions comprised of compound stimuli (e.g., Name some red things) for a 6-year-old child with autism. The intraverbal prompts involved providing the function, feature, and class of the target responses. A multiple probe across behaviors design was used. Results indicated that the child's total number of divergent responses was increased and maintained during two-week follow-up trials. Novel responses were observed across conditions.
\end{abstract}

Keywords: intraverbal prompts, thematic prompts, divergent multiple control, response diversity, autism. 
Using Intraverbal Prompts to Increase Divergent Intraverbal Responses by a Child with Autism

The defining characteristics in autism spectrum disorders (ASD) include impaired social communication as well as restrictive, repetitive patterns of activities and interests (American Psychiatric Association, 2013). It is commonly observed that individuals with ASD often engage in stereotyped behavior and repetitive communication in various contexts, causing impediments in their social and adaptive functioning. Predicaments resulting from invariant patterns of behaviors include the loss of opportunities for meaningful social interactions, limited skills acquisition, and obtaining reinforcement in generalized environments (Rodriguez \& Thompson, 2015; Wolfe, Slocum, \& Kunnavatana, 2014). Consequently, individuals with ASD are often described as "inflexible," "rigid," "stubborn," or the ones with a "strong personality."

Response variability is demonstrated as an operant behavior and can be increased or decreased through the manipulation of contingencies along with prompting procedures in various types of behaviors (Neuringer, 2002, 2004). Empirical research on the acquisition of response variability is particularly relevant in remediation of key deficits resulting from restrictive, repetitive patterns of interest and behavior associated with ASD. Furthermore, variability or novelty of responses is recognized as a key element of generalization (Stokes \& Baer, 1977; Stokes \& Osnes, 2016), required for problem solving, and potentially associated with creativity (Neuringer, 2002). One important type of behavior requiring varied responses pertains to answering others' questions.

In the operant analysis of verbal behavior, responding to questions is referred as an intraverbal behavior, defined as a form of verbal behavior in which a verbal antecedent stimulus evokes another verbal response that does not have point-to-point correspondence to its preceding stimulus (Skinner, 1957). Some other examples, besides answering questions of 
intraverbal relations, include singing a song, reciting a poem, and social conversations. The convergent multiple control refers to multiple stimuli controlling for one response, while the divergent multiple control refers to a single stimulus controlling multiple responses (Michael, Palmer, \& Sundberg, 2011; Skinner, 1957). These two types of multiple control are frequently observed in intraverbal relations. For example, a math word problem that provides several clues to seek one final answer is an example of convergent control. Divergent multiple control is in effect when responding to creativity tasks that require multiple responses to a single question. For typically developing children, acquiring functional intraverbal relations can probably be accomplished through natural contingencies along with maturation; however, the same developmental milestone may not easily be reached for children with ASD if these behaviors are not taught specifically through systematically planned instructional sequences (Michael et al., 2011; Sundberg \& Sundberg, 2011).

Applied operant-based researchers have developed several effective instructional strategies to increase the number and variability in responses to one single task or question for individuals with ASD who exhibit undesired rote intraverbal or repetitive patterns. Henson (1975) used tokens as reinforcement to increase the number of divergent responses to categorical questions for middle school students with different abilities, including students who were gifted, average, and with developmental disabilities. Goetz and Baer (1973) also used social reinforcement to differentially increase novel forms of block building for typical preschoolers. Several studies found that lag schedules of reinforcement (reinforcing only novel responses differing from certain number of preceding responses) effectively increased response variability in answering social questions for children with ASD (Lee, McComas, \& Jawor, 2002; Susa \& Schlinger, 2012). In these studies, the use of reinforcement to increase response variability required target behavior already existing in the individuals' repertoires, if not; researchers had to establish those target behaviors prior to implementation. In addition, 
two types of behavior regarding response variability were targeted. The first type involved divergent multiple control with one stimulus evoking multiple responses (Goetz \& Baer, 1973; Henson, 1975), while the second type did not involve divergent control but with one stimulus evoking only one response (Lee et al., 2002; Susa \& Schlinger, 2012).

Transfer of stimulus control procedures (e.g., echoic to intraverbal, textual to intraverbal, tact to intraverbal) have been effective in increasing the total number of varied responses to categorical questions for individuals with ASD and other developmental disabilities (Braam \& Poling, 1983; Feng, Chou, \& Lee, 2017; Goldsmith, LeBlanc, \& Sautter, 2007; Ingvarsson \& Hollobaugh, 2011; Ingvarsson \& Le, 2011). Although the transfer of stimulus control procedures were effective in establishing multiple responses to single questions, their effects on response variability were not clear. That is, the individuals under training may have acquired desired rote responses to obtain reinforcement without having to provide novel responses. In addition, the echoic and textual prompts were formal prompts with point-to-point correspondence between stimuli and responses that may develop response-form prompt dependency in some of the individuals who rely solely on direct repetition of desired responses without attending to the antecedent stimuli.

Carroll and Kodak (2015) found that instructive feedback provided in conjunction with verbal praise for correct responses as consequences effectively increased response variability for two children with ASD. This teaching strategy eliminated the possibility of prompt dependence while effectively increasing varied responses to categorical questions. It is also relatively easy to implement in clinical settings. However, a ceiling was set for a total of three responses to each categorical question (e.g., "Tell me three animals"). It is possible that total number of varied responses to each target question was inhibited due to the fact that the children were asked to provide three answers or the rote pattern of three answers had been established in the history of reinforcement. 
In the beginning of instruction, it may be helpful to establish a desired rote pattern of responding, particularly in some basic skills. However, such a pattern should be advertently avoided in order to build up response diversity for more advanced skill sets, such as categorical questions. One possible option is to use intraverbal prompts (or thematic prompts, Skinner, 1957) without formal similarity or point-to-point correspondence to the target responses. Sundberg, Endicott, and Eigenheer (2000) used a specific form of intraverbal prompts (i.e., Sign ___ ) to establish signed tacts for two children with ASD who had difficulty acquiring tact through standard training (i.e., "What is this?"). Intraverbal prompts have also been used to teach problem solving strategies for advanced learners, such as typically developing children, to increase the number of categorical intraverbal responses (Sautter, LeBlanc, Jay, Goldsmith, \& Carr, 2011). Specifically, the children were intraverbally prompted to apply the rules of associating category with subcategory (animal-ocean) and then subcategory with items (ocean-whales, dolphins, seals) when answering categorical questions ("Tell me some animals"). In this case, the intraverbal prompts comprised of rules required convergent multiple control to aggregate compound stimuli in the rules and then the divergent control to generate multiple responses in order to solve the given categorical questions. The use of intraverbal prompts to teach varied responses of categorical questions to children with ASD has not been investigated.

The purpose of the present study was to provide preliminary evidence with a clinical demonstration of using intraverbal prompts to increase the total number of varied responses to categorical questions for a child with ASD, who was observed to engage in repetitive and restricted patterns of activities and interests. Each categorical question involved a compound stimulus with two components (e.g., red things), as defined by Eikeseth and Smith (2013). The intraverbal prompts consisted of the function, feature, and class (FFC) of the target responses. The convergent control was required in which the child had to conditionally 
discriminate three stimuli of FFC and evoke a particular response. The divergent control was also required that the total number of varied responses (no ceiling) was targeted. The research question was whether such intraverbal prompts could effectively increase the number of divergent responses to categorical questions involving compound stimuli.

\section{Method}

\section{Participant}

A 6-year-old boy, Chow, served as the participant in the study. Chow was diagnosed with high-functioning autism by a physician with clinical observations based on diagnostic criteria of Diagnostic and Statistical Manual of Mental Disorders IV (DSM-IV) (American Psychiatric Association, 1994), the Chinese version of Clancy Autism Behavior Scale (Clancy, Dugdalei, \& Rendle-Shortt, 1969), and his IQ score (IQ = 88), measured by the Chinese version of Wechsler Intelligence Scale for Children IV (Wechsler, 2003). At the time of the study, he attended a full time kindergarten day program in a general education classroom for half day and a center-based behavioral intervention program for children with autism for two hours per day, one day per week. Chow was selected as a participant for this study because he was observed to engage in restrictive and repetitive patterns of interest when having conversations with others. His intraverbal responses were limited to one specific answer to a single question without variability. His parents and school teachers were concerned about his restrictive patterns in his daily routines and inability to adjust or change planned activities when unexpected events occurred. As a result, Chow often engaged in solitary activities in school.

Chow had verbal and non-verbal functional communication skills to indicate his needs and wants. He used full sentences when making requests. He followed multiple-step directions when each step in the direction was given individually. He responded to his name when his teacher called him individually but not in a group. He answered basic social 
questions but most of them were invariant responses. He was observed to engage in solitary play activities without any interactions with peers. When required to participate in a group activity, he often did not follow group rules and engaged in solitary activities. His cognitive abilities included labeling more than 100 objects (i.e., body parts, actions, animals, food, places, furniture, and vehicles) and their function, feature and class. He also read Chinese at the average of the first grade level. During the time of this study, Chow also had other social programs, such as identifying others' emotions within social contexts, asking "WH" questions, participating in group play activities, and appropriate play skills.

\section{Setting}

The behavioral treatment center is affiliated with a university located in a major city in Taiwan. The official language is Mandarin, and therefore, the instructional programs were conducted in Mandarin. All sessions were conducted in the classroom of the treatment center the child attended. The classroom is 8 by $2.7 \mathrm{~m}$ in area, and $2 \mathrm{~m}$ floor to ceiling. It has three partitioned teaching areas $(2.25 \times 1.35 \mathrm{~m}$ each $)$ for one-on-one instruction and a play area with toys and books on shelves. Each teaching area contained a table with two chairs, a token board on table, a VCR, and two cabinets to organize teaching materials. The sessions were conducted in one of the partitioned areas, with the presence of two other children and their one-on-one instructors in the classroom.

\section{Experimental Design}

A multiple probe design across behaviors (Cooper, Heron, \& Heward, 2007) was used to examine the effects of the intraverbal prompt procedure on the acquisition of divergent intraverbal responses to questions involving objects in five different colors (e.g., "Tell me red things").

The sequence of the study is as follows, (a) baseline probe trials for all target categorical questions, (b) intervention on categorical questions in the order of green, yellow, and red 
color of objects and a probe trial on the target question prior to intervention each day, (d) baseline probe trials for subsequent target categorical questions, and (e) two-week maintenance probe trials following the mastery of each target categorical question.

\section{Response Definitions and Dependent Measures}

The terminal goal was to increase the total number of divergent responses to each target categorical question. Divergent responses refer to correct multiple varied responses each time one single categorical question was asked. A correct response was defined as an independent intraverbal response in accordance with the given question within three seconds upon hearing the question without any prompts. Three types of correct responses were recorded in this study: taught responses, novel responses, and previously emitted untaught responses. A taught response refers to the child providing one of the target items taught with intraverbal prompts during intervention. A novel response was defined as an independent intraverbal response to a given question that was not emitted or taught previously. A previously emitted untaught response refers to an independent response that was not taught by the instructor but was provided previously. Thus, when an untaught response was provided for the first time, it was scored as a novel response, but if the same response was provided after the first time, it was scored as a previously emitted untaught response. An incorrect response was defined as an irrelevant answer to the target question or no response within 3 seconds upon hearing the question. To conclude a trial, the instructor would ask the child whether he had more answers (e.g., "anything more?") until the child indicated no more answers.

\section{Interobserver Agreement and Procedural Integrity}

All sessions were videotaped for the purpose of assessing interobserver agreement and procedural integrity. To assess interobserver agreement, another graduate student serving as a second observer was trained prior to the study to record student responses independently and separately with the instructor during the sessions. Point-to-point agreement was checked 
immediately at the conclusion of the trial. If any disagreements occurred, both the instructor and the observer would watch the video to check the student's responses. Therefore, the accuracy of data on the student's responses for each trial was assured. The instructor and the observer then coded the responses as taught responses, novel responses, and previously emitted untaught responses. The data on agreement of three types of responses were $100 \%$.

The procedural integrity was assessed by a supervisor who used a checklist containing components of antecedent and consequence specified for responses, including the correct delivery of questions, the correct implementation of 3-second time delay, the correct delivery of intraverbal prompts when the student had no responses, the correct reinforcement delivered when student provided a correct response, the correct delivery of correction procedure when the student did not give an accurate answer, and a correct final check to conclude the trial. The procedural integrity was assessed for $30 \%$ of the total sessions evenly distributed across conditions. The data on procedural integrity was $100 \%$ for all sessions observed.

\section{Procedure}

Baseline probe trials. All target questions were tested to obtain the initial level of performance. For example, the child was asked "Tell me some things that are red," and "Name some red things, as many as you can." In all of the baseline probe trials, the instructor allowed the participant a maximum of 10 seconds to respond and then would ask the student, “Any more answers?" to confirm that the student has no more answers. (The student typically answered, "No more," or "I don't know.”) The experimenter provided social praises for each correct response and encouraged the child to provide more answers, while ignoring incorrect responses. If the child did not respond within 10 seconds, the instructor would ask, “Anything?" If the child indicated no answers, the instructor would provide a general social praise for good attending behaviors. 
Intervention. As preparation for the intervention, the instructor pre-selected 25 target items to be taught for each target question as the terminal goal (See Table 1 for the list of selected target items used in this study). These items were selected from the child's known items and tested to ensure that the child accurately tacted their function, feature, and class (FFC).

The student had a token board on his table and was offered tokens on a variable schedule throughout the 30-minute individual instructional sessions. A probe trial on the target question under intervention was conducted to assess the student's acquisition in the beginning of each individual session. The training trials for categorical questions with intraverbal prompts were interspersed with the child's other instructional programs.

In the beginning of intervention, the instructor selected five target items from the list of 25 target items (Table 1) as a short-term goal and began a training trial by presenting the target question and waiting for a maximum of 3 seconds for the child to respond. If the child provided no response or paused for 3 seconds after one response, the instructor provided an intraverbal prompt involving one of the target items' FFC. For example, an intraverbal prompt for a red apple as a red thing involved 'Guess what? You can eat this thing. It's yellow inside and is a fruit." If the child said, "a red apple," the instructor immediately provided social praises. If the child gave an inaccurate answer, no response within three seconds, or responded with, "I don't know," the instructor informed him the correct answer, "It's a red apple!" After consequation for the response was complete, the instructor waited for another three seconds and presented the intraverbal prompt for the next target item, if no response occurred. The same sequence was repeated for each target item until all five target items were presented in a random order. A training trial was then completed. Specifically, one training trial consisted of a) the target question (e.g., "Tell me red things, as many as you can"), b) a three-second time delay, c) child response, d) consequation, e) repeating b) to d) 
for all five target items. A total of three training trials were presented during the 30-minute individual instructional session each day.

As the intervention progressed, the child began to emit multiple correct responses without breaks. When this occurred, the instructor did not interrupt with praises but nodded with smiles and delivered tokens in a variable schedule. At the end of the child's multiple responses, the instructor either provided an intraverbal prompt for a target item not mentioned by the child, or delivered social praises to conclude a training trial when the child included all five target items. Specific social praises concerning the novelty of responses and tokens were delivered to reinforce the occurrences of novel responses. However, if the child provided an incorrect response or multiple incorrect responses along with other correct responses, the instructor waited until the child paused, delivered praises specific to each correct response and a correction for each incorrect response (e.g. "A taxi is yellow, not red.”). In this study, Chow did not provide any incorrect answers during training trials. He typically indicated that he did not have any more answers.

To avoid rote memorization, the following steps were implemented within or in-between training trials throughout the study. First, the target questions were asked in various forms (e.g., "Tell me some red stuff," or "Name things that are red" or "What things are red?") Second, the child's other instructional programs were interspersed between training trials. Third, the intraverbal prompts of five selected target items were presented in a random sequence. Finally, if the child was observed to provide rote chained responses (e.g., stating the same responses in the same sequence repeatedly for two consecutive trials), the instructor provided an intraverbal prompt upon delivering the target question to evoke a different response sequence.

The mastery criterion for each pre-selected target item taught under intervention was achieved when the child independently emitted the target response during a probe trial for 
two consecutive days. Once a target item reached the criterion, the intraverbal prompt for such an item was no longer presented, and a new item from the list of 25 target items was added to the pool of five target items as a short-term goal. When all 25 target items reached the mastery criterion, the intervention for the target question ended.

Follow-up maintenance probe trials. The follow-up maintenance probe trials were conducted two weeks after the mastery criterion was achieved for each target question. These probe trials were conducted with an identical procedure as the baseline probe trials. No practice opportunities for target questions occurred after the completion of the intervention.

\section{Results}

Figure 1 depicts Chow's responses for each target categorical question during probe trials across all conditions. During the first baseline probe trials, Chow provided two answers for green and red things, four for yellow things, one for orange things, and zero for purple things. He maintained the identical two answers for green things during subsequent baseline probe trials. As the intervention was introduced, his total responses for green things during probe trials gradually increased from a low level to a high level and then maintained at the mid-level until the end of the intervention. He also emitted a total of 13 novel responses during probe trials under the intervention condition. He maintained divergent responses for green things at a high level with one novel response in the follow-up probe trial. See Table 1 for the novel responses.

Baseline data of yellow things and red things indicated that Chow's responses to these two target questions ranged from four to eight responses prior to the introduction of intervention. Under the intervention condition, the total divergent responses gradually increased from five to 13 responses in both yellow and red things, with one and 10 novel responses for yellow things and red things, respectively. Chow also maintained at a mid-level in the follow-up probe trials. 
For orange things and purple things, he provided one response for orange things and no response for purple things during initial baseline probe trials. The total responses increased to four and five responses for orange things and purple things during subsequent baseline probe trials while the second target question (yellow things) was undergoing the intervention. The intervention for orange and purple things was not introduced due to the age of the child which made him ineligible to continue the services.

The percentage of non-overlapping data (PND) of probe trials between baseline and intervention conditions is $100,73.68$, and 72.72 for green things, yellow things, and red things, respectively. The PND between baseline and follow-up conditions was 100 for all three target categorical questions.

\section{Discussion}

The present study was a preliminary investigation of using intraverbal prompts containing FFC to increase the total number of divergent responses to categorical questions with compound stimuli. This serves as a clinical demonstration of a new teaching tactic - the intraverbal prompts to increase the total number of divergent responses. The results indicated that the intraverbal prompts with FFC of the target items were potentially effective in increasing total divergent responses to categorical questions involving compound stimuli for a child with ASD. The PND effect sizes between baseline and follow-up conditions also indicated a fairly large post-intervention effect for all three target categorical questions. .

The use of an object's FFC for prompting provides instructional assistance necessary for successful acquisition while avoiding possible prompt dependence or restrictive, repetitive patterns of responding to prompts with point-to-point correspondence. The intraverbal prompts with FFC also offer opportunities to facilitate convergent control and conditional discriminations in the child's repertoire. In addition, intraverbal prompts are relatively easy to implement in clinical settings, compared to tact prompts or lag schedules of 
reinforcement.

The child's divergent responses to categorical questions were emerging during initial baseline probe trials but increased to a high level for all three target questions following the completion of the intervention. While the first target question was undergoing intervention, the total number of divergent responses for the second and subsequent target questions during baseline increased from their initial probe trials, indicating the occurrence of stimulus generalization of divergent response to similar but different categorical questions. It was possible that the divergent control acquired from the first target question was generalized to the subsequent target questions due to the history of reinforcement on similar categorical questions. However, the total number of divergent responses emitted during baseline remained at a relatively low level, compared to the total responses provided in probe trials during and after the intervention. The relatively high level of responses after intervention suggested the effectiveness of the intervention. Although the occurrence of such generalization posits a lack of experimental control in this case, the behavioral change is of clinical significance. It is important that future researchers employ an experimental design that includes generalization probe trials to assess stimulus generalization or use thematically and topographically different questions as target questions to obtain better experimental control (Osnes \& Lieblein, 2003).

The continued generation of novel responses to the categorical questions under intervention and follow-up conditions indicated that FFC intraverbal prompts not only increased the total number of divergent responses but also the novel responses. Further, novel responses were still provided occasionally in later trials under intervention or follow-up conditions when generating novel responses were more challenging. The occurrence of response generalization was potentially from following multiple sources. First, the verbal stimulus "as many as you can" that required divergent control may function as a rule for the 
child to emit divergent responses. Second, the reinforcement provided to divergent responses as well as novel responses strengthened response generalization. Third, the diversity of target items for each question ( 25 pre-selected items) provided sufficient exemplars. Fourth, the FFCs used as prompts during intervention are easy to find in any setting outside of training, making generalization more likely to occur. The components of the intervention contained some critical features necessary for generalization to occur (Stokes \& Baer, 1977; Stokes \& Osnes, 2016). To further investigate this important topic, researchers may teach children to use FFC intraverbal prompts as a rule to solve problems in complex categorical questions with compound stimuli and test the generalization effects on newly combined compound stimuli. In addition, future studies could also compare the efficacy and efficiency of different instructional methods (e.g., formal prompts, thematic prompts, instructional feedback) in increasing divergent responses for children with ASD.

The results of this study were consistent with previous applied studies that children with ASD could acquire divergent responses and/or response variability in intraverbal relations (Rodriguez \& Thompson, 2015; Wolfe et al., 2014). This particular intervention not only addressed the key deficit of restrictive and repetitive interests/behaviors displayed by children with ASD but also teach them multiple control in intraverbal relations. It is necessary for future researchers to evaluate various effects of intraverbal prompts with FFC or compare other types of prompts on the acquisition of divergent responses for questions involving compound stimuli. Besides comparing the intervention effects of different instructional tactics, it is also imperative to examine convergent and divergent control in intraverbal relations and to specify multiple control in the instructional procedure as well as the outcome measures under investigation.

In this study, each target question was a compound stimulus containing a color in objects. The child was required to provide as many answers as he could to each question without any 
limits. During baseline probe trials, the child responded to each question with one response and relied on the experimenter to verbally prompt for more answers (e.g., “Anything else?”). Restrictively speaking, divergent control was not in effect during baseline because the child only responded with one answer to a single verbal stimulus. As the intraverbal prompts were introduced, the child began to provide multiple answers without needing any verbal prompts for more.

Although the child had included many identical answers for each target question from earlier probe trials to the later ones, these answers did not appear in the same sequence each time. We only observed rote responses during initial training trials but attempted to interrupt the sequence in the middle of the child's recitation by inserting one or two answers in a random order. Therefore, such a response interruption strategy may be necessary during initial training. We did not observe a rote response pattern with an invariant sequence of responses for any target questions during probe trials in this study. Future studies should examine the effects of adding such an interruption procedure on the total number of divergent responses or on the novel responses.

Limitations of this study included the lack of experimental control in a multiple probe across behaviors design, incomplete intervention for the fourth and fifth target questions, limited number of participants, limited use of compound stimuli, and inconsistent use of tokens contingent upon novel responses during baseline and intervention sessions. We only implemented target questions involving five different colors in conjunction with objects but not any other discriminative stimuli or recombination of them. It is important to incorporate different combinations of compound stimuli to strengthen convergent control in the intraverbal relations under investigation. The delivery of tokens under a continuous schedule upon novel responses under the intervention condition but not during baseline may also had an effect on the occurrences of novel responses. That is, the token reinforcement 
during intervention may have inadvertently placed novel responses under a loosely controlled lag 1 schedule of reinforcement. Future experiments should determine a consistent reinforcement schedule during baseline and training sessions in order to isolate the effects of intraverbal prompts.

Despite the limitations, the results of the present study had important implications in the educational intervention of complex intraverbal relations for children with ASD. The intervention aimed at teaching divergent control to increase the number of responses to categorical questions. The novel responses were also increased collaterally. The design of the intervention also incorporated important components to facilitate generalization. The FFC intraverbal prompts required convergent control for a particular response thus avoiding the child's engaging in an undesired rote pattern of responding or simple direct repetitions. Such prompts can also be used in the initial stage of establishing response diversity or in the later stage to strengthen or increase response diversity in an already established pattern. Additionally, the FFC intraverbal prompts were also relatively easy for practitioners to implement in the applied settings without constant monitoring for occurrences of novel responses or reinforcement schedules. 


\section{References}

American Psychiatric Association (Ed.). (1994). Diagnostic and statistical manual of mental disorders: DSM-IV. Washington, DC.: Author.

American Psychiatric Association. (2013). Diagnostic and statistical manual of mental disorders (DSM-5®). Washington, D. C.: Author.

Braam, S. J., \& Poling, A. (1983). Development of intraverbal behavior in mentally retarded individuals through transfer of stimulus control procedures: classification of verbal responses. Applied Research in Mental Retardation, 4(4), 279-302.

Carroll, R. A., \& Kodak, T. (2015). Using instructive feedback to increase response variability during intraverbal training for children with autism spectrum disorder. The Analysis of Verbal Behavior, 31(2), 183-199. https://doi.org/10.1007/s40616-015-0039-x

Clancy, H., Dugdalei, A., \& Rendle-Shortt, J. (1969). The diagnosis of infantile autism. Developmental Medicine \& Child Neurology, 11(4), 432-442. https://doi.org/10.1111/j.1469-8749.1969.tb01461.x

Cooper, J. O., Heron, T. E., \& Heward, W. L. (2007). Applied behavior analysis (2nd ed). Upper Saddle River, N.J: Pearson/Merrill-Prentice Hall.

Eikeseth, S., \& Smith, D. P. (2013). An analysis of verbal stimulus control in intraverbal behavior: Implications for practice and applied research. The Analysis of Verbal Behavior, 29(1), 125-135.

Feng, H., Chou, W.-C., \& Lee, G. T. (2017). Effects of tact prompts on acquisition and maintenance of divergent intraverbal responses by a child with autism. Focus on Autism and Other Developmental Disabilities, 32(2), 133-141. https://doi.org/10.1177/1088357615610540

Goetz, E. M., \& Baer, D. M. (1973). Social control of form diversity and the emergence of 
new forms in children's blockbuilding. Journal of Applied Behavior Analysis, 6(2), 209-217. https://doi.org/10.1901/jaba.1973.6-209

Goldsmith, T. R., LeBlanc, L. A., \& Sautter, R. A. (2007). Teaching intraverbal behavior to children with autism. Research in Autism Spectrum Disorders, 1(1), 1-13. https://doi.org/10.1016/j.rasd.2006.07.001

Henson, F. O. (1975). An investigation of the effects of token reinforcement on divergent verbal responding. Journal of Applied Behavior Analysis, 8(4), 459. https://doi.org/10.1901/jaba.1975.8-459

Ingvarsson, E. T., \& Hollobaugh, T. (2011). A comparison of prompting tactics to establish intraverbals in children with autism. Journal of Applied Behavior Analysis, 44(3), 659-664. https://doi.org/10.1901/jaba.2011.44-659

Ingvarsson, E. T., \& Le, D. D. (2011). Further evaluation of prompting tactics for establishing intraverbal responding in children with autism. The Analysis of Verbal Behavior, 27(1), 75-93.

Lee, R., McComas, J. J., \& Jawor, J. (2002). The effects of differential and lag reinforcement schedules on varied verbal responding by individuals with autism. Journal of Applied Behavior Analysis, 35(4), 391-402. https://doi.org/10.1901/jaba.2002.35-391

Michael, J., Palmer, D. C., \& Sundberg, M. L. (2011). The multiple control of verbal behavior. The Analysis of Verbal Behavior, 27(1), 3-22.

Neuringer, A. (2002). Operant variability: Evidence, functions, and theory. Psychonomic Bulletin \& Review, 9(4), 672-705.

Neuringer, A. (2004). Reinforced variability in animals and people: Implications for adaptive action. American Psychologist, 59(9), 891-906. https://doi.org/10.1037/0003-066X.59.9.891

Osnes, P. G., \& Lieblein, T. (2003). An explicit technology of generalization. The Behavior 
Analyst Today, 3(4), 364-374.

https://doi.org/http://dx.doi.org.proxy2.cl.msu.edu/10.1037/h0099994

Rodriguez, N. M., \& Thompson, R. H. (2015). Behavioral variability and autism spectrum disorder. Journal of Applied Behavior Analysis, 48(1), 167-187. https://doi.org/10.1002/jaba.164

Sautter, R. A., LeBlanc, L. A., Jay, A. A., Goldsmith, T. R., \& Carr, J. E. (2011). The role of problem solving in complex intraverbal repertoires. Journal of Applied Behavior Analysis, 44(2), 227-244. https://doi.org/10.1901/jaba.2011.44-227

Skinner, B. F. (1957). Verbal behavior. New York: Appleton-Century-Crofts.

Stokes, T. F., \& Baer, D. M. (1977). An implicit technology of generalization. Journal of Applied Behavior Analysis, 10(2), 349-367. https://doi.org/10.1901/jaba.1977.10-349

Stokes, T. F., \& Osnes, P. G. (2016). An operant pursuit of generalization - republished article. Behavior Therapy, 47(5), 720-732. https://doi.org/10.1016/j.beth.2016.08.012

Sundberg, M. L., Endicott, K., \& Eigenheer, P. (2000). Using intraverbal prompts to establish tacts for children with autism. The Analysis of Verbal Behavior, 17, 89-104.

Sundberg, M. L., \& Sundberg, C. A. (2011). Intraverbal behavior and verbal conditional discriminations in typically developing children and children with autism. The Analysis of Verbal Behavior, 27(1), 23-43.

Susa, C., \& Schlinger, H. D. (2012). Using a lag schedule to increase variability of verbal responding in an individual with autism. The Analysis of Verbal Behavior, 28(1), $125-130$.

Wechsler, D. (2003). Wechsler Intelligence Scale for Children ${ }^{\circledR}$ - Fourth Edition. San Antonio, TX: Psychological Corporation.

Wolfe, K., Slocum, T., \& Kunnavatana, S. (2014). Promoting behavioral variability in individuals with autism spectrum disorders: A literature review. Focus on Autism and 
Other Developmental Disabilities, 29(3), 180-190.

https://doi.org/10.1177/1088357614525661 
Table 1.

Pre-selected target items for each categorical question and the novel responses provided by the child in this study

\begin{tabular}{|c|c|}
\hline Green Things & $\begin{array}{l}\text { trees, leaves, grass, bamboo, forests, clover, } 4^{\text {th }} \text { color in rainbows, } \\
\text { Hillside, broccoli, pea, rice plant, bok choy, spinach, cabbage, } \\
\text { loofah guard, asparagus, cucumber, bamboo viper snakes, } \\
\text { chameleons, frogs, Christmas trees, green mark environmental } \\
\text { signs, green traffic lights, postman uniforms, postal vehicles }\end{array}$ \\
\hline $\begin{array}{l}\text { Novel responses } \\
(\mathrm{n}=16)\end{array}$ & $\begin{array}{l}\text { Guavas, sugar apples, lettuce, sweet potato leaves, string beans, } \\
\text { scallion, stick insects, okra, mung beans, chive, spinach, turtle } \\
\text { Trees*, leaves*, forests*, grass* }\end{array}$ \\
\hline Yellow Things & $\begin{array}{l}\text { Sun, sunflowers, 3rd color in rainbows, cheese, corn, yellow bell } \\
\text { peppers, lemons, pears, yellow melons, golden kiwi, banana, key } \\
\text { limes, chicks, canaries, ducklings, gold python, McDonald signs, } \\
\text { taxi, yellow traffic lights, school buses, warning signs, crookneck } \\
\text { squash, butter, egg yolks, sponge Bob }\end{array}$ \\
\hline $\begin{array}{l}\text { Novel responses } \\
(\mathrm{n}=13)\end{array}$ & $\begin{array}{l}\text { durian, star fruits, bananas*, key limes*, golden kiwi*, } 3^{\text {rd }} \text { color } \\
\text { in rainbows*, pears*, taxi*, sun*, egg yolks*, yellow bell } \\
\text { peppers*, canaries* }\end{array}$ \\
\hline Red Things & $\begin{array}{l}\text { blood, maple leaves, poinsettias, } 1^{\text {st }} \text { color in rainbows, tongues, } \\
\text { lips, ketchup, chili sauce, chili peppers, red bell peppers, beets, } \\
\text { wax apples, strawberries, tomatoes, raspberries, cherries, red } \\
\text { apples, African red dragonflies, blood parrot cichlid, traffic light, } \\
\text { watermelon, fire engine, Chinese wedding flowers, Chinese New } \\
\text { Year couplets, red envelopes }\end{array}$ \\
\hline $\begin{array}{l}\text { Novel responses } \\
(\mathrm{n}=22)\end{array}$ & $\begin{array}{l}\text { tamarisk, cockscomb, red seaweed, fire extinguisher, firecracker, } \\
\text { Alishan train, 7-11 sign, tomato sauce, Chinese wedding gowns } \\
\text { red apples*, strawberries*, } 1^{\text {st }} \text { color in rainbows*, red traffic } \\
\text { lights*, watermelons*, fire engines*, blood*, chili peppers*, wax } \\
\text { apples*, cherries*, tomatoes*, red bell peppers*, beets* }\end{array}$ \\
\hline
\end{tabular}

Note. Items with an asterisk represent novel responses provided by the child identical to the pre-selected target items. 


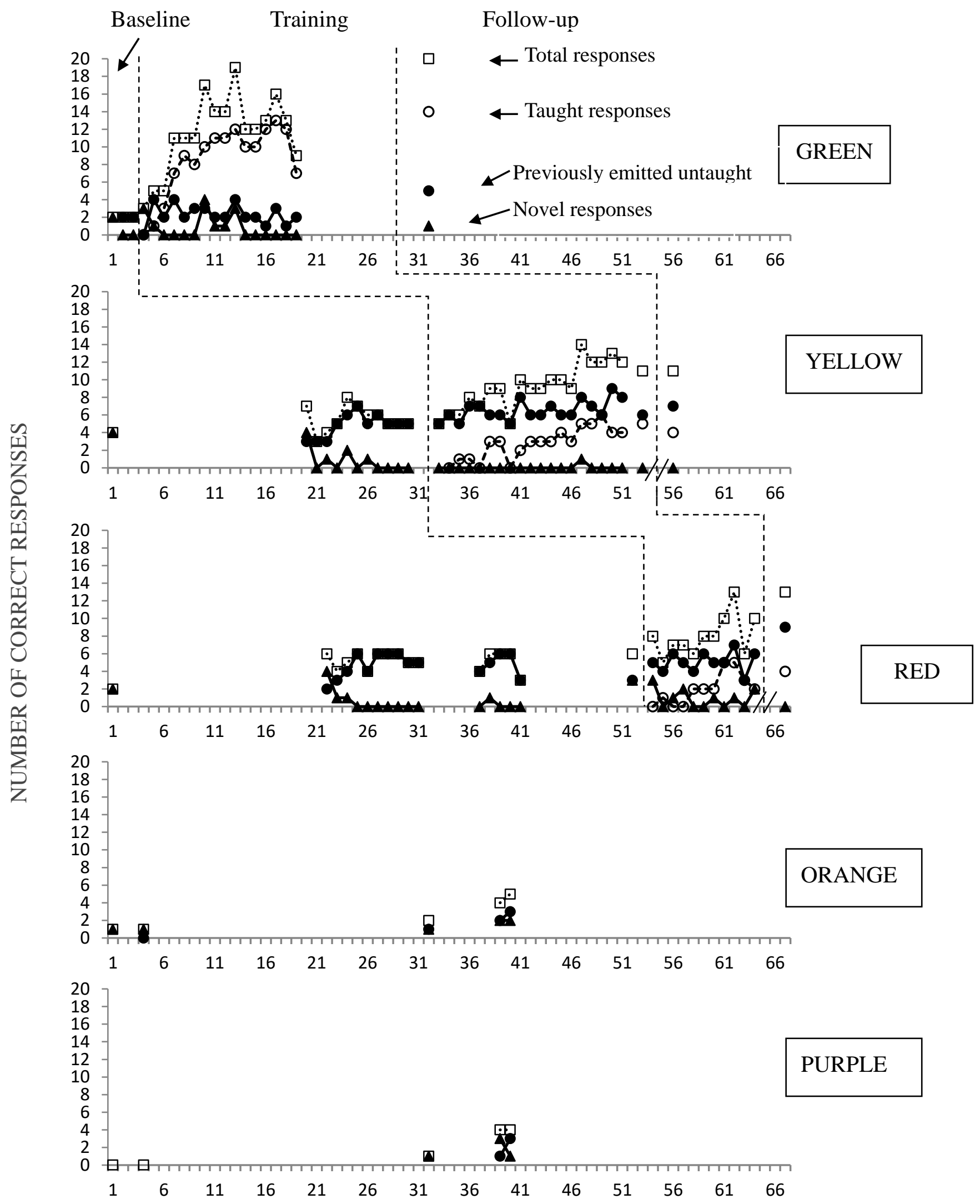

Figure 1. Chow's responses during probe sessions for all target questions across all conditions. 